Géographie physique et Quaternaire

\title{
La dépression de Claye-Souilly-Saint-Denis : nouvelles données sur les réorganisations hydrographiques en région parisienne (France)
}

\section{The Claye-Souilly-Saint-Denis depression : new data on drainage pattern modifications in the Paris region (France)}

\section{Jean-Pierre Larue}

Volume 60, numéro 2, 2006

URI : https://id.erudit.org/iderudit/016824ar

DOI : https://doi.org/10.7202/016824ar

Aller au sommaire du numéro

Éditeur(s)

Les Presses de l'Université de Montréal

ISSN

0705-7199 (imprimé)

1492-143X (numérique)

Découvrir la revue

Citer cet article

Larue, J.-P. (2006). La dépression de Claye-Souilly-Saint-Denis : nouvelles données sur les réorganisations hydrographiques en région parisienne (France). Géographie physique et Quaternaire, 60(2), 119-129.

https://doi.org/10.7202/016824ar
Résumé de l'article

Cette étude tente d'expliquer la formation de la dépression topographique qui relie la vallée de la Marne à celle de la Seine, entre Claye-Souilly et Saint-Denis. L'analyse sédimentologique des dépôts quaternaires présents dans la dépression indique la prédominance des apports locaux alluviaux et colluviaux, mais la présence de minéraux lourds d'origine volcanique implique des retombées éoliennes et des remaniements aux dépens des alluvions de la Seine et de la Marne. La position topographique et la taille des minéraux excluent la présence d'alluvions en place de la Seine et de la Marne, mais suggèrent plutôt des remaniements par les affluents. Le creusement de la dépression est antérieur à la mise en place des alluvions des hautes terrasses et à la capture de la Beuvronne par la Marne. Les effondrements karstiques ont également joué un rôle important dans le façonnement de la dépression de Claye-Souilly-Saint-Denis. 


\section{LA DÉPRESSION DE CLAYE- SOUILLY-SAINT-DENIS: NOUVELLES DONNÉES SUR LES RÉORGANISATIONS HYDROGRAPHIQUES EN RÉGION PARISIENNE (FRANCE)}

Jean-Pierre LARUE*; Géodynamique des milieux naturels et de l'environnement, Université de Paris XII-Val de Marne, 61 avenue du Général de Gaulle, 94010 Créteil Cedex, France.

RESUME Cette étude tente d'expliquer la formation de la dépression topographique qui relie la vallée de la Marne à celle de la Seine, entre Claye-Souilly et Saint-Denis. L'analyse sédimentologique des dépôts quaternaires présents dans la dépression indique la prédominance des apports locaux alluviaux et colluviaux, mais la présence de minéraux lourds d'origine volcanique implique des retombées éoliennes et des remaniements aux dépens des alluvions de la Seine et de la Marne. La position topographique et la taille des minéraux excluent la présence d'alluvions en place de la Seine et de la Marne, mais suggèrent plutôt des remaniements par les affluents. Le creusement de la dépression est antérieur à la mise en place des alluvions des hautes terrasses et à la capture de la Beuvronne par la Marne. Les effondrements karstiques ont également joué un rôle important dans le façonnement de la dépression de ClayeSouilly-Saint-Denis.
ABSTRACT The Claye-Souilly-Saint-Denis depression: new data on drainage pattern modifications in the Paris region (France). This work tries to explain the formation of the depression which links the Marne valley to the Seine valley, between Claye-Souilly and SaintDenis. Sedimentological analysis of the Quaternary deposits located in the depression shows that local alluvial and colluvial sediments are predominant, but the presence of volcanic heavy minerals testifies of aeolian deposits and reworking of the Seine and Marne alluvia. The altitude and the grain-size of the deposits exclude in situ alluvial deposits of the Seine and the Marne, but propose reworking of the deposits by the tributaries. The depression was excavated before the accumulation of high terraces and the Beuvronne capture by the Marne. Karstic subsidence has also played an important part in the genesis of the Claye-Souilly-Saint-Denis depression. 


\section{INTRODUCTION}

Depuis Davis (1895), l'intérêt des géomorphologues pour l'étude des réorganisations hydrographiques ne s'est guère démenti. La recherche des traces morphologiques de capture a été complétée par l'analyse sédimentologique des dépôts conservés, permettant de reconstituer les profils longitudinaux des paléotracés successifs (Weisrock, 1997). Les études récentes insistent sur les impacts géomorphologiques à l'amont et à l'aval des captures (Mather, 2000; Maher et al., 2007). Ces recherches ont révélé la complexité des mécanismes et des causes des réorganisations hydrographiques: par exemple, la capture de la Moselle est expliquée par des facteurs généraux, tectoniques, climatiques et morphodynamiques, auxquels s'ajoutent des facteurs locaux comme les diffluences karstiques (Harmand et al., 1995; Losson et Quinif, 2001). Néanmoins, les processus précis des changements de cours sont souvent mal élucidés (Bishop, 1995). C'est le cas dans le Bassin parisien où la plupart des captures ont été repérées, mais où leurs mécanismes continuent à être discutés (Tricart, 1952; Harmand et al., 1995; Larue et Étienne, 1997 ; Pastre et Leroyer, 1997 ; Losson et Quinif, 2001). Dans la région parisienne, les changements de tracé de la Seine et de la Marne demeurent mal connus malgré de nombreux travaux (Chaput, 1923; Cailleux, 1943; Lécolle, 1989; RoblinJouve, 1995 ; Le Béchennec et al., 1998). Aussi, l'étude détaillée de la dépression de Claye-Souilly-Saint-Denis peutelle contribuer à mieux comprendre la formation et l'évolution d'une vallée fossile qui résulte de l'interaction entre plusieurs processus géomorphologiques.

Au nord et au nord-est de Paris, cette dépression topographique, d'une longueur de $22 \mathrm{~km}$, relie d'est en ouest la vallée de la Marne à celle de la Seine (fig. 1). Étroite à l'amont (250 m à Claye-Souilly) et à l'aval (moins de $1 \mathrm{~km}$ à SaintDenis), elle s'étend sur plus de $5 \mathrm{~km}$ dans le secteur de Sevran-Bobigny. La ligne de hauteurs qui la délimite au sud, de Montmartre à Lagny, est interrompue par les couloirs de Rosny-sous-Bois et de Gagny-Villemomble qui la relient à la vallée de la Marne. Drainée par le réseau du Crould à l'ouest et par celui de la Beuvronne à l'est, cette dépression, marécageuse dans la zone de partage des eaux, présente un fond plat dans lequel s'encaissent faiblement les cours d'eau actuels. Les altitudes du fond diminuent graduellement vers l'ouest: $65 \mathrm{~m}$ à l'ouest de Claye-Souilly, $55 \mathrm{~m}$ à Sevran et $45 \mathrm{~m}$ à Bobigny.

Les strates sédimentaires du centre du Bassin parisien présentent un pendage faible vers le sud, ce qui a permis le dégagement de plateformes structurales (Soyer, 1953; Pomerol, 1967; Pomerol et Feugueur, 1974; Diffre, 1980). Le fond de la dépression est modelé dans les marnes à gypse et les sables de Monceau bartoniens sus-jacents au calcaire de Saint-Ouen qui donne au nord la plateforme structurale de la Plaine de France (fig. 2). Au sud, les buttes de l'interfluve allant de Montmartre à Lagny, forment la terminaison des plateformes de Brie et de Beauce (calcaires et meulières du Stampien inférieur). La partie occidentale de la dépression correspond à l'ondulation synclinale de la fosse de Saint-Denis encadrée par le prolongement de l'anticlinal du Bray, au nord- est, et par l'anticlinal Meudon-Saint-Maur, au sud-ouest (Cavelier et Lorenz, 1987).

Cette morphologie suggère une vallée fossile qui aurait pu être façonnée par la Beuvronne avant sa capture par la Marne (Dollfus, 1903; Caudron et al., 1971), mais également par la Seine ou le Crould (Roblin-Jouve, 1995). Un paléotracé de la Marne dans la dépression de Claye-Souilly-St-Denis a été envisagé par Pastre et Leroyer (1995), puis abandonné par ces mêmes auteurs (Pastre et Leroyer, 1997). En outre, le dégagement de cette dépression a pu être favorisé par la dissolution du gypse qui engendre périodiquement des effondrements spectaculaires (Dollfus, 1903; Hirschauer, 1991). La dépression comprend trois ensembles morphologiques: les fonds de vallée vers $50 \mathrm{~m}$ d'altitude, une terrasse en forme de replat vers $60 \mathrm{~m}$ d'altitude et la présence d'un glacis vers $75 \mathrm{~m}$ d'altitude sur le versant nord du massif de l'Aulnay (fig. 2).

Les dépôts quaternaires qui tapissent la dépression ont été classés différemment selon les auteurs. Caudron et al. (1971), Diffre (1980), Roblin-Jouve (1995) distinguent les alluvions récentes $\left(F_{z}\right)$ des plaines d'inondation des cours d'eau et les dépôts anciens $\left(F_{y}\right)$ des terrasses. Hirschauer (1991) cartographie trois ensembles principaux qui présentent des faciès différents: les dépôts des lits majeurs en amont, ceux des basses vallées situées entre Dugny et Saint-Denis et ceux des «pseudo-terrasses » qui couvrent les plaines de Bondy, Drancy, La Courneuve et Bobigny. Si la mise en place des alluvions récentes est bien connue (Roblin-Jouve, 1995; Le Béchennec et al., 1998; Lafarge, 1998), celle des dépôts anciens demeure très hypothétique.

Pour tenter d'expliquer la genèse de cette dépression, nous utiliserons principalement l'étude sédimentologique des formations détritiques conservées et en particulier l'analyse des minéraux lourds pour déterminer les sources des matériaux et séparer les apports des différents cours d'eau. Une fois les formations bien caractérisées, leur raccordement de l'amont à l'aval permet de préciser la chronologie de leur mise en place et des changements de cours.

\section{ANALYSE DES FORMATIONS DÉTRITIQUES CONSERVÉES}

\section{DONNÉES DE TERRAIN}

Ces dépôts ont pu être étudiés à partir des sondages et forages répertoriés par le Bureau de Recherches Géologiques et Minières (BRGM), à la faveur de tranchées comme à l'ouest de Claye-Souilly, de fouilles archéologiques comme La Vache à l'aise à Bobigny et grâce aux sondages effectués par les Ponts et Chaussées dans le bois de la Tussion à Sevran, suite aux effondrements survenus en 1999. Les dépôts quaternaires, d'une épaisseur variant entre 1 et $8 \mathrm{~m}$, sont constitués de sables fins ocres à gris, plus ou moins argileux et sont presque toujours dépourvus de galets, ce qui les différencie des alluvions de la Seine et de la Marne qui en contiennent toujours. Leur limite inférieure est parfois délicate à fixer lorsqu'ils entrent en contact avec les sables de Monceau éocènes qui présentent des faciès très semblables. À Livry-Gargan, 


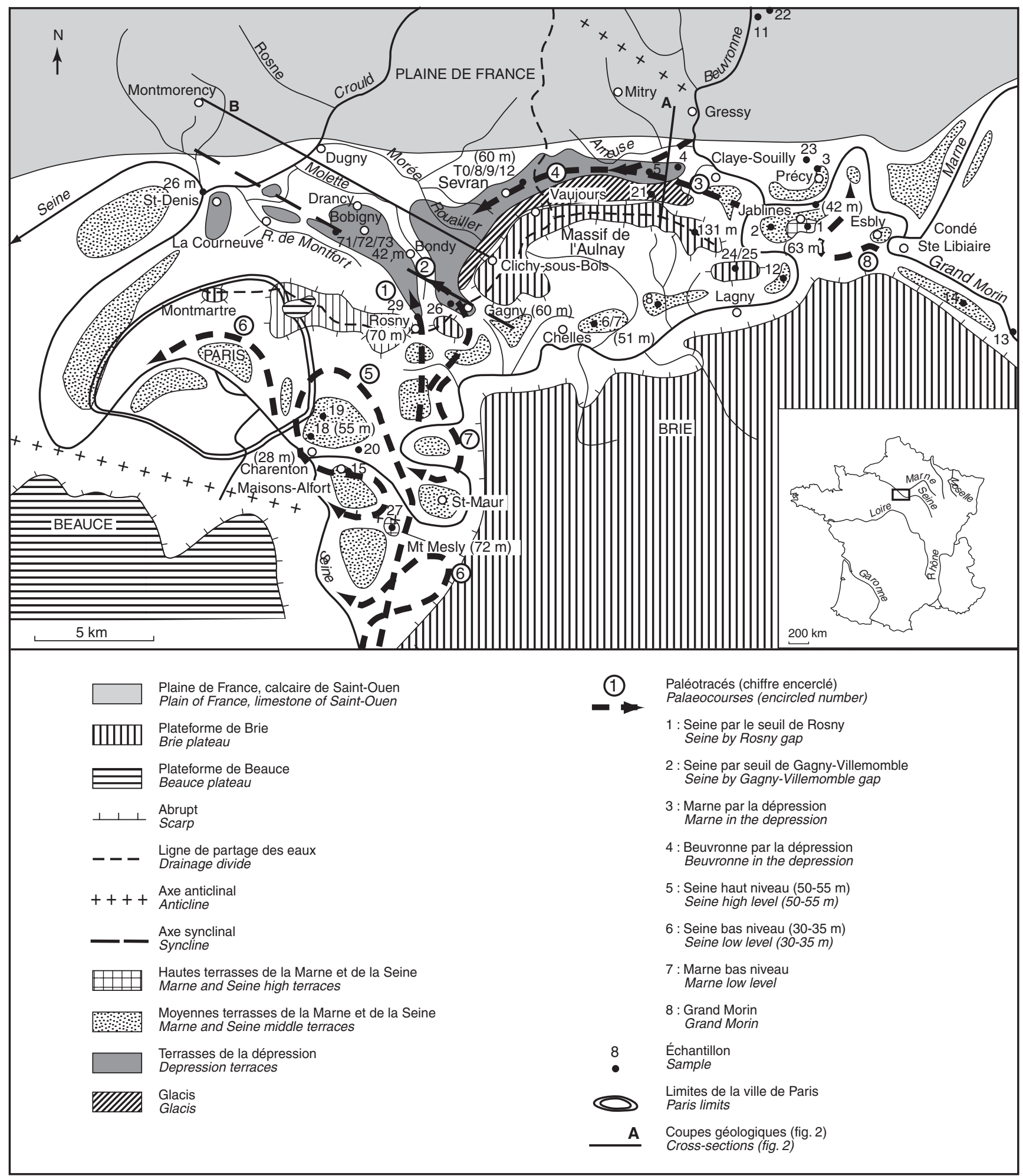

FIGURE 1. Carte de localisation de la dépression de Claye- Location map of the Claye-Souilly-Saint-Denis depression. Souilly-Saint-Denis. 
les coupes établies lors de l'entretien du lac de Sévigné et de l'étang de Verchain, situés dans la vallée supérieure du Rouailler, montrent que les colluvions du versant nord-ouest du massif de l'Aulnay sont ravinées par $8 \mathrm{~m}$ de sables grossiers à moyens qui forment une terrasse de $63 \mathrm{~m}$ d'altitude. Les granulométries indiquent des alluvions bien classées de 0,3 $\mathrm{mm}$ de diamètre médian (Hirschauer, 1991).

Des échantillons, présentés au tableau I et sur la figure 1, ont pu être prélevés sur le site archéologique de La Vache à l'aise $(71,72,73)$ et dans les carottes de sondage du Bois de la Tussion (T0, T8, T9, T12). Les coupes du premier site (Roblin-Jouve, 1995) montrent, du bas vers le haut, sur environ 2,50 $\mathrm{m}$ : (1) des sables et des limons blanc-jaune (2,5 YR $8 / 3$ ) disposés en grandes rides obliques présentant des concrétionnements d'alios et des rubéfactions, (2) des sables argileux blancs à galets calcaires, (3) des sables et des argiles stratifiés, (4) des sables jaunes à taches grises et à langues jaunes, puis un sol gris et le sol noir actuel. L'échantillon 71 provient du niveau 1, les échantillons 72 et 73 , du niveau 4 . Les sondages du Bois de la Tussion montrent des dépôts dont l'épaisseur varie de 2 à $5 \mathrm{~m}$ et présentent tous la lithostratigraphie suivante: des sables fins à moyens à la base, des marnes plus ou moins limoneuses au milieu et des limons café au lait (7,5 YR 4/4) au sommet, puis le sol actuel. Les quatre échantillons sont issus de la strate inférieure qui est aussi la plus sableuse. De plus, d'autres échantillons ont été prélevés, à la faveur de terrassements urbains, d'affleurements et de sablières, dans les dépôts des seuils de Rosnysous-Bois et de Gagny-Villemomble, dans les alluvions des vallées actuelles de la Marne, de la Seine, du Grand Morin et de la Beuvronne, dans le substrat sédimentaire (sables de Monceau, de Beauchamp, de Mortefontaine, de Fontainebleau), dans les limons sommitaux et dans les dépôts de glacis (fig. 1 et tabl. I) afin de pouvoir comparer les matériaux et déterminer l'origine des dépôts de la dépression.

\section{MÉTHODOLOGIE}

Les échantillons ont subi l'analyse granulométrique et morphoscopique, la diffractométrie des rayons $\mathrm{X}$ et la détermination des minéraux lourds. Le granulomètre laser LS Coulter permet de classer les particules comprises entre 0,375 et $2000 \mu \mathrm{m}$, ce qui couvre la totalité des fractions sableuses, limoneuses et argileuses. Les courbes cumulatives bi-logarithmiques à ordonnées de probabilité font apparaître les écarts de distribution par rapport à une distribution gaussienne, ce qui permet d'individualiser les différents stocks granulométriques (Visher, 1969). Concentrés selon la méthode densimétrique de séparation au bromoforme (Parfenoff et al., 1970), les minéraux lourds ont été identifiés au microscope optique. Les pourcentages de minéraux transparents (tabl. II) ont été calculés à partir de l'analyse d'au moins 200 grains. Les résultats chiffrés ont été soumis à l'analyse factorielle des correspondances (AFC) afin de réaliser une discrimination objective des échantillons. Les poudres de la fraction $<40 \mu \mathrm{m}$ ont été soumises à la diffractométrie des rayons $X$ afin de déterminer les minéraux argileux qui fournissent des données sur l'origine des sédiments et sur l'altération qu'ils ont subie
(Moore et Reynolds, 1997). Les spectres ont été réalisés avec un diffractomètre de poudre muni d'une sonde à compteurs à scintillation, pour la raie $\mathrm{K}$ alpha du cuivre.

\section{RÉSULTATS}

Les courbes granulométriques (fig. 3) montrent des sédiments sablo-limoneux, où des différences sont observables entre le Bois de la Tussion et Bobigny. Les sédiments du Bois de la Tussion donnent deux modes: le premier se situe entre 350 et $430 \mu \mathrm{m}$ et le second vers $45 \mu \mathrm{m}$ alors que ceux de Bobigny n'en possèdent qu'un entre 430 et $480 \mu \mathrm{m}$. Mieux classés, les dépôts de Bobigny sont aussi plus grossiers que ceux de Sevran: la médiane est plus forte à Bobigny $(466 \mu \mathrm{m})$ qu'à Sevran (325 $\mu \mathrm{m})$, la teneur en argiles est beaucoup plus faible à Bobigny $(0,22 \%)$ qu'à Sevran (entre 1,12 et $3,39 \%$ ). Révélatrice du milieu de dépôt, l'asymétrie est positive à Sevran et négative à Bobigny. L'asymétrie négative s'explique par l'évacuation des éléments fins par le courant. Les courbes bi-logarithmiques indiquent que les dépôts du Bois de la Tussion résultent d'un transport en suspension et d'un dépôt par décantation, alors que ceux de Bobigny comportent en plus un stock transporté par saltation et même par roulage.

La morphoscopie révèle une majorité $(75 \%)$ de grains émoussés et mats pour les particules supérieures à $200 \mu \mathrm{m}$ et environ $90 \%$ de grains non-usés pour les fractions fines inférieures à $100 \mu \mathrm{m}$. Ces résultats distinguent ces dépôts des alluvions de la Seine et de la Marne analysées par Cailleux (1943): les fractions sableuses de $700 \mu \mathrm{m}$ de la Seine possèdent $24 \%$ de grains non-usés, $55 \%$ d'émoussésluisants et $21 \%$ de ronds-mats, celles de la Marne, $63 \%$ de non-usés, $18 \%$ d'émoussés-luisants et $19 \%$ de ronds-mats.

Les spectres diffractométriques révèlent des minéraux argileux très peu abondants: les pics d'illite et de kaolinite peu marqués témoignent d'une faible altération in situ. La silice domine la calcite dans tous les échantillons, y compris ceux des terrasses de la Marne et de la Seine. Cependant, à Sevran et Bobigny, on y trouve de la sépiolite et de la palygorskite qui individualisent ces dépôts par rapport aux alluvions de la Seine et de la Marne. Ces minéraux argileux peuvent provenir du calcaire de Saint-Ouen qui en renferme.

L'analyse des minéraux lourds (tabl. II) ajoute des précisions sur l'origine des dépôts de la dépression. Les sédiments tertiaires sont caractérisés, (1) par un faible nombre d'espèces minérales (6 à 10), (2) par la prédominance nette, soit de la tourmaline pour les sables de Beauchamp (éch. 23), soit du zircon pour les sables de Monceau (éch. 20), de Mortefontaine (éch. 22) et de Fontainebleau (éch. 25), (3) par un faible pourcentage de staurotide et (4) par l'absence de minéraux volcaniques. Cette composition les sépare des dépôts de la dépression qui possèdent une composition minéralogique plus variée (14 à 16 espèces minérales), sont plus riches en staurotide et renferment toujours des éléments d'origine volcanique. En revanche, les limons sommitaux (24), les dépôts de glacis (21) et les alluvions présentent de fortes similitudes avec les dépôts de la dépression. Les alluvions de la Beuvronne (11) renferment du grenat et de la hornblende 

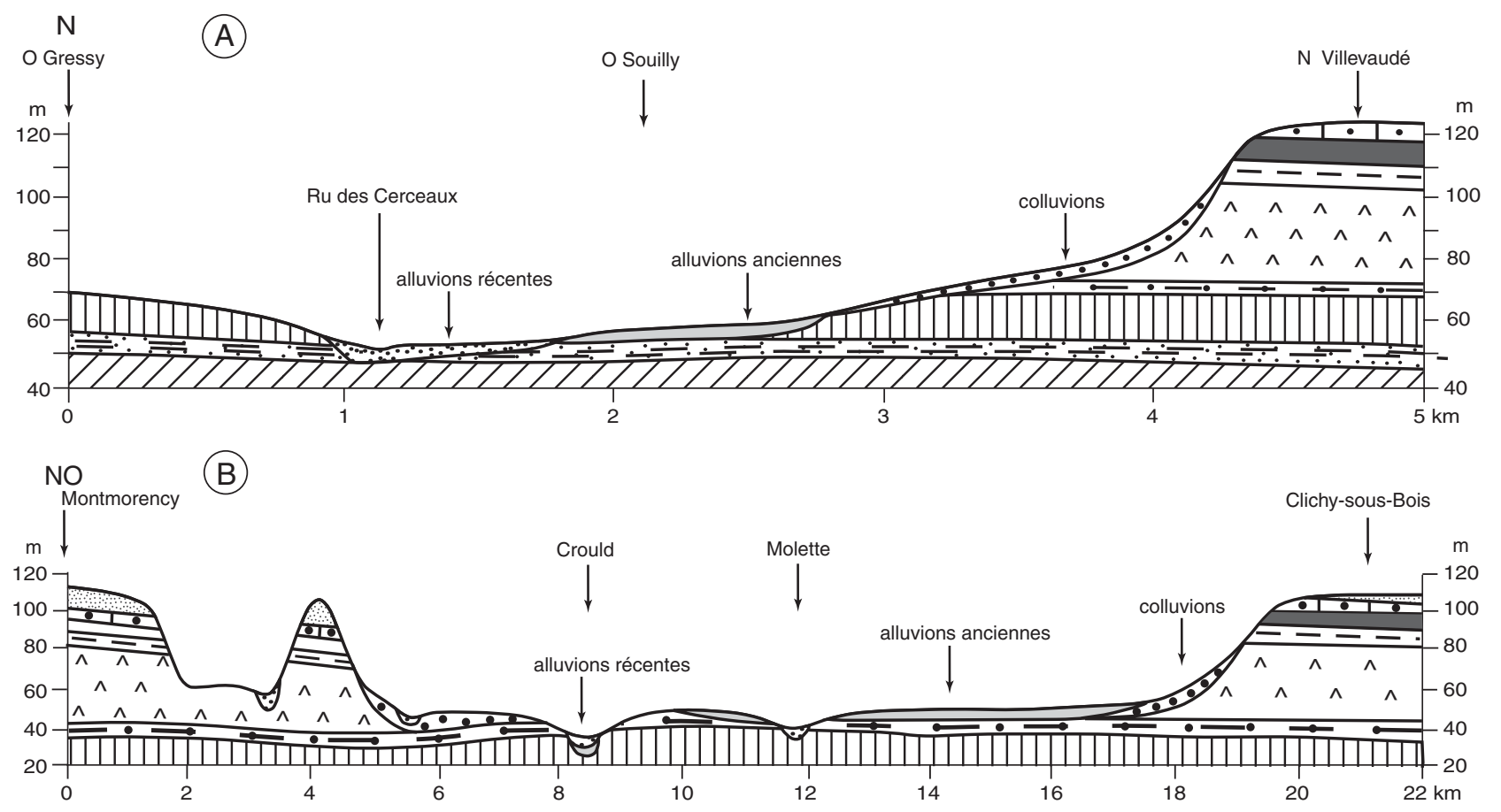

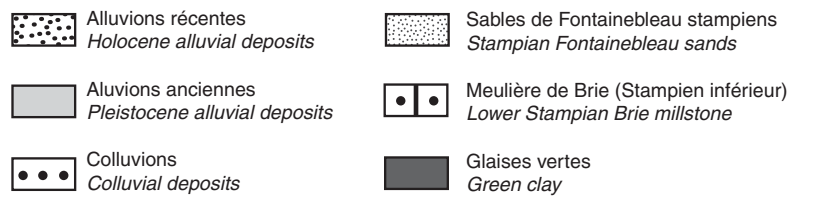

FIGURE 2. Coupes géologiques de la dépression de ClayeSouilly-Saint-Denis: (A) à l'ouest de Claye-Souilly et (B) au droit de Bobigny, entre Montmorency et Clichy-sous-Bois.

verte que l'on retrouve dans les dépôts de la partie orientale de la dépression. Les alluvions de la Seine $(15,16,17,18$, 27 ) et de la Marne $(1,2,3,6,7,8)$ présentent de fortes teneurs en staurotide. Les dépôts conservés sur les seuils de Gagny-Villemomble (26) et de Rosny (29) ont une composition peu différente des alluvions anciennes de la Seine, où la présence d'augites émoussées supérieures à $400 \mu \mathrm{m}$ de diamètre témoigne de leur transport fluviatile depuis le Massif central (Tourenq et Pomerol, 1995; Larue, 2003).

Les dépôts de la dépression n'ont cependant pas une composition homogène. Ainsi, les teneurs en minéraux volcaniques varient verticalement et latéralement: à Bobigny, elles atteignent $30 \%$ dans les sables supérieurs et seulement $7 \%$ dans les sables inférieurs, à Sevran, elles fluctuent entre 1 et $4 \%$. À Bobigny, le zircon domine la tourmaline dans les sables inférieurs (71), alors que l'inverse se produit pour les dépôts supérieurs $(72,73)$. La composition des premiers est assez peu différente de celle des alluvions de la haute terrasse de la Seine. La présence d'orthose signalée par Roblin-Jouve (1995) renforce cette similitude. À Bobigny et à Villemomble, les apports fluviatiles lointains sont attestés par la découverte d'augites très émoussées supérieures à $400 \mu \mathrm{m}$ de diamètre, provenant du volcanisme du Massif central (Pastre et Cantagrel, 2001). Les alluvions de la moyenne terrasse de la Marne renferment quelques augites de taille inférieure à $200 \mu \mathrm{m}$, mais sont dépourvues de sphène et de hornblende, alors que celles de la Seine contiennent les trois minéraux.

Pour la partie orientale de la dépression, il apparaît que les dépôts pourraient provenir d'un mélange entre des alluvions de la Beuvronne et des apports colluviaux issus du versant du massif de l'Aulnay. Pour le secteur de Bobigny, les apports locaux sont mêlés à des alluvions plus grossières pouvant être issues du remaniement d'alluvions de la Seine déposées antérieurement.

\section{INTERPRÉTATION}

\section{ORIGINE ET ÂGE DES DÉPÔTS DE LA DÉPRESSION}

De par leur altitude, les dépôts tapissant la partie orientale de la dépression se situent dans le prolongement de la haute terrasse de la Marne conservée à Jablines à $63 \mathrm{~m}$ (soit $20 \mathrm{~m}$ d'altitude relative par rapport au lit mineur actuel de la Marne) et absente plus en aval (fig. 4). Ils ne peuvent appar- 
TABLEAU I

Localisation et lithostratigraphie des échantillons prélevés

\begin{tabular}{|c|c|c|c|c|c|c|}
\hline Site & Lieu & Carte $1 / 50000$ & $\begin{array}{c}\text { Coordonnées } \\
\text { Lambert }\end{array}$ & $\begin{array}{l}\text { Altitude } \\
(\mathrm{m})\end{array}$ & $\begin{array}{l}\text { Profondeur } \\
\quad(\mathrm{m})\end{array}$ & Lithostratigraphie \\
\hline 1 & SE Jablines & Lagny & $638,8-134,7$ & 63 & 0,6 & Sable argileux $\left(F_{x}\right)$ \\
\hline 2 & SO Jablines & Lagny & $630,0-135,0$ & 50 & 1 & Sable $\left(F_{y}\right)$ \\
\hline 3 & Préçy-sur-Marne & Lagny & $632,3-137,0$ & 52 & 2 & Sable $\left(F_{y}\right)$ \\
\hline 4 & NO Claye-Souilly & Lagny & $624,0-138,8$ & 60 & 1 & Sable fin ocre $\left(F_{y}\right)$ \\
\hline 5 & S La Platrière & Lagny & $621,8-139,0$ & 56 & 0,5 & Sable argileux $\left(F_{y}\right)$ \\
\hline 6 & E Chelles & Lagny & $620,0-131,0$ & 50 & 1 & Sable fin $\left(F_{y}\right)$ \\
\hline 7 & E Chelles & Lagny & $620,0-131,0$ & 50 & 2 & Sable fin $\left(F_{y}\right)$ \\
\hline 8 & E Brou & Lagny & $622,3-131,4$ & 52 & 1 & Argile sableuse $\left(F_{y}\right)$ \\
\hline 11 & N St-Mesmes & $\begin{array}{l}\text { Dammartin- } \\
\text { en-Goëlle }\end{array}$ & $626,3-143,5$ & 66 & 0,7 & Limon sableux $\left(F_{z}\right)$ \\
\hline 12 & NE Dampmart & Lagny & $631,3-132,8$ & 47 & 2 & Sable $\left(F_{y}\right)$ \\
\hline 13 & S Créçy-la-Chapelle & Coulommiers & $642,0-126,0$ & 50 & Lit majeur & Sable de débordement $\left(F_{z}\right)$ \\
\hline 14 & St-Germain-sur-Morin & Lagny & $637,6-131,6$ & 48 & 1 & Alluvions caillouteuses $\left(F_{y}\right)$ \\
\hline 15 & Maisons-Alfort & Paris & $606,4-123,8$ & 33 & 2 & Alluvions saaliennes \\
\hline 16 & Sannois & Melun & $630,2-86,4$ & 54 & 1 & Alluvions $\mathrm{F}_{\mathrm{x}}$ \\
\hline 17 & Sannois & Melun & $630,0-86,0$ & 63 & 0,8 & Alluvions $\mathrm{F}_{\mathrm{w}}$ \\
\hline 18 & Charenton-le-Pont & Paris & $605,0-125,6$ & 55 & 0,6 & $\begin{array}{l}\text { Sable rouille } \\
\text { (haute terrasse) }\end{array}$ \\
\hline 19 & Lac Daumesnil & Paris & $605,6-125,4$ & 50 & 0,6 & $\begin{array}{l}\text { Argile sableuse } \\
\text { (Sable de Beauchamp) }\end{array}$ \\
\hline 20 & Plateau de Gravelle & Paris & $607,7-124,5$ & 65 & 0,6 & $\begin{array}{l}\text { Sable argileux } \\
\text { (Sable de Monceau) }\end{array}$ \\
\hline 21 & NE Villevaudé & Lagny & $624,2-137,0$ & 75 & 0,5 & Dépôts de glacis \\
\hline 22 & E Nantouillet & $\begin{array}{l}\text { Dammartin- } \\
\text { en-Goëlle }\end{array}$ & $627,5-144,8$ & 80 & 3 & Sable de Mortefontaine \\
\hline 23 & Préçy-sur-Marne & Lagny & $632,0-137,0$ & 53 & 1 & Sable de Beauchamp \\
\hline 24 & N Dampmart & Lagny & $629,0-132,7$ & 122 & 0,5 & Limon \\
\hline 25 & N Dampmart & Lagny & $629,0-132,7$ & 122 & 3 & Sable de Fontainebleau \\
\hline 26 & Villemomble & Paris & $612,5-132,0$ & 60 & 0,5 & Alluvions $\mathrm{F}_{\mathrm{y}}$ \\
\hline 27 & Mont Mesly & Corbeil & $610,0-119,3$ & 72 & 0,6 & Alluvions $\mathrm{F}_{\mathrm{x}}$ \\
\hline 29 & Rosny-sous-Bois & Paris & $611,0-130,8$ & 70 & 0,6 & Alluvions $\mathrm{F}_{\mathrm{y}}$ \\
\hline T0 & Bois de la Tussion & Lagny & $616,2-138,0$ & 60 & 2,5 & Sable limoneux $\left(F_{y}\right)$ \\
\hline T8 & Bois de la Tussion & Lagny & $616,2-138,0$ & 60 & 2 & Sable argileux $\left(F_{y}\right)$ \\
\hline T9 & Bois de la Tussion & Lagny & $616,2-138,0$ & 60 & 2,5 & Sable moyen beige $\left(F_{y}\right)$ \\
\hline T12 & Bois de la Tussion & Lagny & $616,2-138,0$ & 60 & 3,4 & Argile sableuse verte $\left(F_{y}\right)$ \\
\hline 71 & $\begin{array}{l}\text { Bobigny-La Vache à l'aise } \\
\text { (puits 1026) }\end{array}$ & Paris & $605,8-134,9$ & 42 & 1 & Sable ocre $\left(F_{y}\right)$ \\
\hline 72 & Bobigny (fossé 142) & Paris & $605,8-134,9$ & 42 & 1,4 & Sable ocre $\left(F_{y}\right)$ \\
\hline 73 & Bobigny (fossé 242) & Paris & $605,8-134,9$ & 42 & 1 & Sable ocre $\left(F_{y}\right)$ \\
\hline
\end{tabular}


TABLEAU ॥

Composition en minéraux lourds

\begin{tabular}{|c|c|c|c|c|c|c|c|c|c|c|c|c|c|c|c|c|c|c|c|c|}
\hline & Formation & 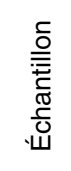 & 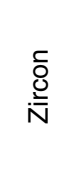 & 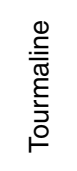 & $\begin{array}{l}\frac{\Phi}{N} \\
\text { Ð } \\
\frac{0}{0} \\
\sum\end{array}$ & 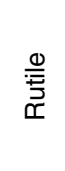 & $\begin{array}{l}\text { 을 } \\
\frac{0}{0} \\
\dot{0}\end{array}$ & 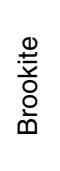 & $\begin{array}{l}\bar{\varpi} \\
\frac{\widetilde{0}}{\mathbb{N}} \\
\mathbb{U}\end{array}$ & $\begin{array}{l}\frac{0}{5} \\
\frac{10}{0} \\
\frac{0}{\frac{10}{0}} \\
\frac{0}{\frac{0}{4}}\end{array}$ & 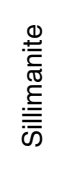 & 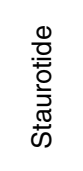 & 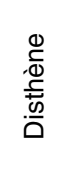 & 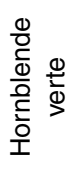 & $\begin{array}{l}\frac{0}{0} \\
\frac{0}{0} \\
\frac{0}{0} \\
\frac{1}{5} \\
\frac{5}{5} \\
\text { 호 }\end{array}$ & $\begin{array}{l}\frac{0}{0} \\
\frac{0}{0} \\
\frac{0}{0}\end{array}$ & $\frac{0}{\frac{0}{5}}$ & $\begin{array}{l}0 \\
\frac{1}{0} \\
\frac{1}{0} \\
\text { की }\end{array}$ & 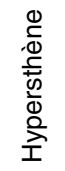 & $\begin{array}{l}\stackrel{0}{N} \\
\stackrel{N}{\frac{1}{0}} \\
\frac{0}{0}\end{array}$ \\
\hline \multirow{9}{*}{ 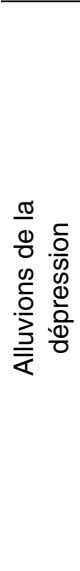 } & $\mathrm{F}_{\mathrm{x}} \mathrm{NO}$ Claye & 4 & 26 & 23 & & 6,5 & & & & 6,5 & & 30 & 2,5 & 2,5 & & & & 2,5 & & \\
\hline & $\mathrm{F}_{\mathrm{x}} \mathrm{O}$ Claye & 5 & 52,5 & 5 & & 4 & & & 1 & 1 & & 24,5 & 5 & & & & 5 & 1 & & \\
\hline & Sevran $(2,5 \mathrm{~m})$ & T0 & 34 & 19,5 & 0,5 & 6,5 & & & 1,5 & 1,5 & & 24,5 & 4 & & & & 4 & & & 1,5 \\
\hline & Sevran $(2,5 \mathrm{~m})$ & T9 & 48 & 13 & & 6 & & 1 & 3,5 & 2 & & 21 & 4 & & & & 1 & & & \\
\hline & Sevran $(3,4 \mathrm{~m})$ & $\mathrm{T} 12$ & 49 & 12,5 & & 9 & & & 2,5 & 1 & & 18,5 & 2,5 & 1 & & & 1 & 3 & & \\
\hline & Puits 1026 (1 m) & 71 & 40 & 19 & & 5,5 & 0,5 & & 3 & 0,5 & 0,5 & 22 & 2 & & & & 5 & 2 & & \\
\hline & Fossé $142(1,4$ m) & 72 & 6,5 & 26 & & & 1 & & 1 & 5,5 & & 23 & 5,5 & & & & 23 & 2 & 5,5 & \\
\hline & Fossé $242(1 \mathrm{~m})$ & 73 & 14,5 & 16 & 1 & 2 & & & 3 & 7 & 0,5 & 15 & 6 & 1,5 & 4 & & 24 & & 2 & 3 \\
\hline & $F_{y}$ Villemomble & 26 & 18,5 & 23 & & 4,6 & & 0,6 & 2 & 0,7 & & 20,5 & 4 & 0,7 & 1,3 & & 3,3 & 11 & & \\
\hline \multicolumn{2}{|c|}{ Dépôts de glacis } & 21 & 71 & 8 & & 3,5 & & 2 & 0,5 & & & 10 & 2,5 & 0,5 & 1 & & 0,5 & 2,5 & & \\
\hline \multicolumn{2}{|c|}{ Limons sommitaux } & 24 & 54,5 & 14,5 & & 3,5 & & & & 2 & & 12,5 & 5,5 & 2 & & & & & & \\
\hline \multirow{7}{*}{ 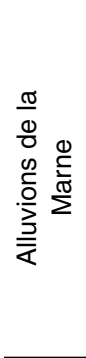 } & $\mathrm{F}_{\mathrm{x}}$ Jablines & 1 & 39 & 23 & & 7 & & 3 & 1 & 1 & 1,5 & 22 & 2,5 & & & & & & & \\
\hline & $\mathrm{F}_{\mathrm{y}}$ Jablines & 2 & 32 & 29 & & 5 & & & & & & 25,5 & 4 & & & 1 & 2,5 & & & \\
\hline & $F_{y}$ Préçy & & 11,5 & 52,5 & 2 & & & & 3 & 3 & 2 & 18 & 5 & & & & 3 & & & \\
\hline & E Chelles (1 m) & 6 & 36,5 & 23,5 & 1 & 4 & & & 2 & 4,5 & & 14 & 4,5 & 5 & & & 1 & 4 & & \\
\hline & E Chelles (2 m) & 7 & 6,5 & 40 & & & & & & 11 & 2 & 37,5 & & & & & 2 & & & \\
\hline & $F_{x} E$ Brou & 8 & 60 & 12 & 1 & 5 & & 1 & & 2 & & 10 & 6 & & & & 2 & & & \\
\hline & $\mathrm{F}_{\mathrm{x}}$ Dampmart & 12 & 65 & 6 & & 5 & & & 1,5 & 1,5 & 0,5 & 15 & 3 & 4 & & & 0,5 & 2 & & \\
\hline \multicolumn{2}{|c|}{$\mathrm{F}_{\mathrm{z}}$ Beuvronne } & 11 & 28 & 33 & & 4 & & & 7 & 4 & & 15 & 4 & 7 & & & & & & \\
\hline \multicolumn{2}{|c|}{$F_{z}$ Grand Morin } & 13 & 40 & 21 & & 5,5 & & 2 & 5,5 & 3,5 & 1 & 12 & 3,5 & 5,5 & & & & & & \\
\hline \multicolumn{2}{|c|}{$\mathrm{F}_{\mathrm{x}}$ Grand Morin } & 14 & 43,5 & 26 & 1 & 8,5 & & & & 2,5 & & 10,5 & 4 & & & & & 3,5 & & \\
\hline
\end{tabular}




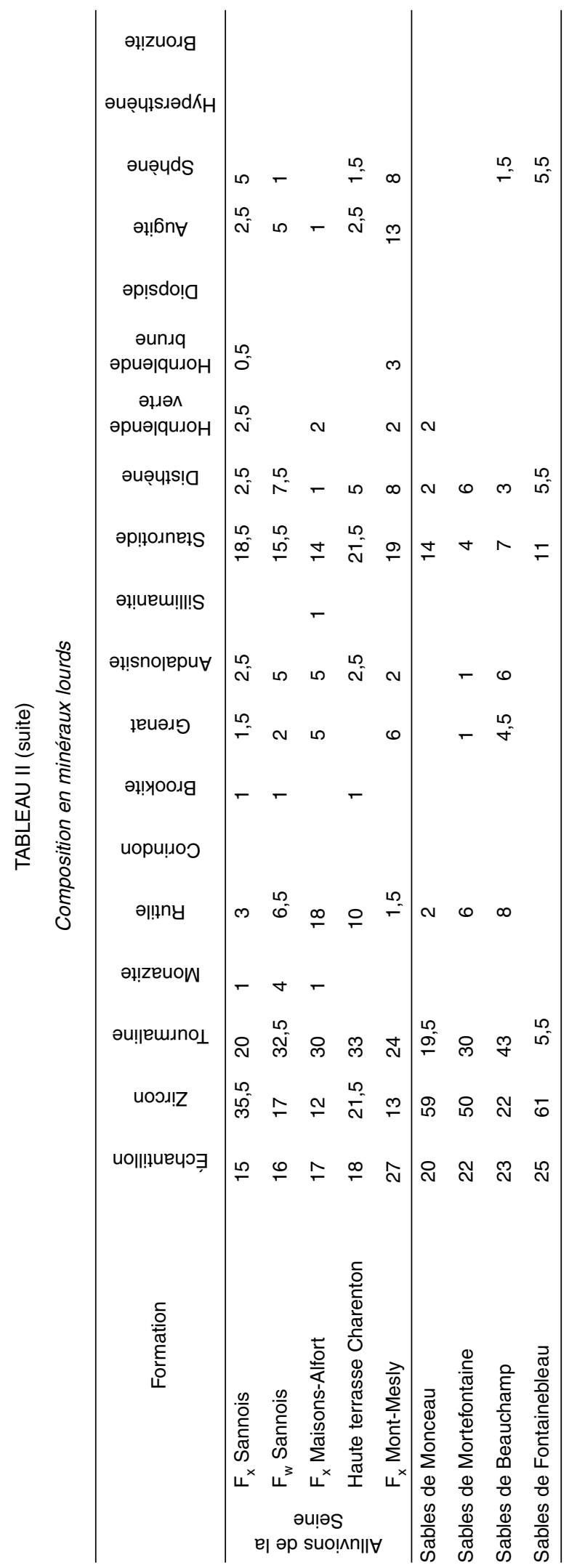

tenir aux nappes formant les terrasses inférieures qui jalonnent la vallée actuelle de la Marne entre Jablines et la confluence avec la Seine (fig. 1). Ils sont donc plus anciens que les alluvions de Chelles placées entre le Mindel et le Würm par la découverte de deux faunes, l'une froide à Elephas primigenius, l'autre chaude à Elephas antiquus (Bourdier, 1969). Rapportées au Riss par Michel (1972), ces terrasses sont composées de plusieurs nappes alluviales qui forment, soit des glacis alluviaux, soit une terrasse de 10$15 \mathrm{~m}$ d'altitude relative. Dans la Région parisienne, RoblinJouve (1980), Rodriguez (1994) et Roblin-Jouve et Rodriguez (1997) ont distingué trois phases d'incision: au Saalien, le substrat est creusé jusqu'à l'étiage actuel et l'accumulation, datée à Maisons-Alfort de $162000 \pm 9000$ et $206500 \pm$ 16500 BP (Durbet et al., 1997), atteint 6 à 10 m d'altitude relative; au Weichsélien, le fond rocheux est incisé de 5 à $10 \mathrm{~m}$ au dessous de l'étiage actuel et le remblaiement suivant culmine à $7 \mathrm{~m}$ d'altitude relative; enfin le creusement tardiglaciaire qui débute au Bølling atteint $10 \mathrm{~m}$ sous l'étiage actuel. D'épais limons sableux recouvrent le fond de la vallée. Pastre et al. (1997) montrent un dispositif semblable dans la vallée de la Marne au droit de Jablines, dans la zone de passage du TGV-Interconnection. Pastre et Leroyer (1995) émettent l'hypothèse d'une capture de la Marne par le Grand Morin au Pléistocène moyen (antérieur au stade isotopique 6 ou 8): le cours aval de la Marne correspondrait à l'ancien cours aval du Grand Morin et les dépôts de la dépression jalonneraient l'ancien cours de la Marne avant la capture réalisée dans le secteur de Jablines. Les minéraux lourds du Grand Morin $(13,14)$ ne confirment toutefois pas cet ancien tracé. En effet, dépourvues d'augite, les alluvions du Grand Morin contiennent également moins de staurotide que celles de la Marne.

Pour le secteur de Bobigny, des apports de la Seine pouvaient passer par les seuils de Rosny vers $70 \mathrm{~m}$ d'altitude ou de Gagny-Villemomble vers $60 \mathrm{~m}$ d'altitude et situés dans le prolongement de la très haute terrasse du Mont Mesly (72 m d'altitude). La présence d'augite émoussée dans ces deux seuils atteste du passage d'une paléo-Seine au début de l'incision des cours d'eau au Pléistocène inférieur. En effet, les principales émissions pyroclastiques du Mont Dore contenant des clinopyroxènes se sont produites entre 2,6 et 1,5 Ma (Pastre et Cantagrel, 2001), soit au tout début de l'encaissement des cours d'eau. En revanche, les alluvions peu altérées de Bobigny, situées seulement à $42 \mathrm{~m}$ d'altitude, n'appartiennent pas à cette nappe, mais elles peuvent inclure des éléments remaniés de cette dernière.

La finesse des matériaux et l'absence de galets distinguent les dépôts de la dépression des alluvions périglaciaires de la Marne et de la Seine (Cailleux, 1943), mais pourraient en faire des alluvions interglaciaires ou tardiglaciaires (Leroyer et al., 1997). La présence d'orthose et de minéraux volcaniques rapproche les sables inférieurs de Bobigny des matériaux transportés par la Seine. Hadjouis (2001) signale la présence de minéraux lourds volcaniques dans les alluvions saaliennes, weichséliennes et tardiglaciaires de la Seine, à Maisons-Alfort. Toutefois, en raison des apports éoliens, l'augite n'est pas spécifique à la Seine puisqu'on en trouve dans les alluvions de 


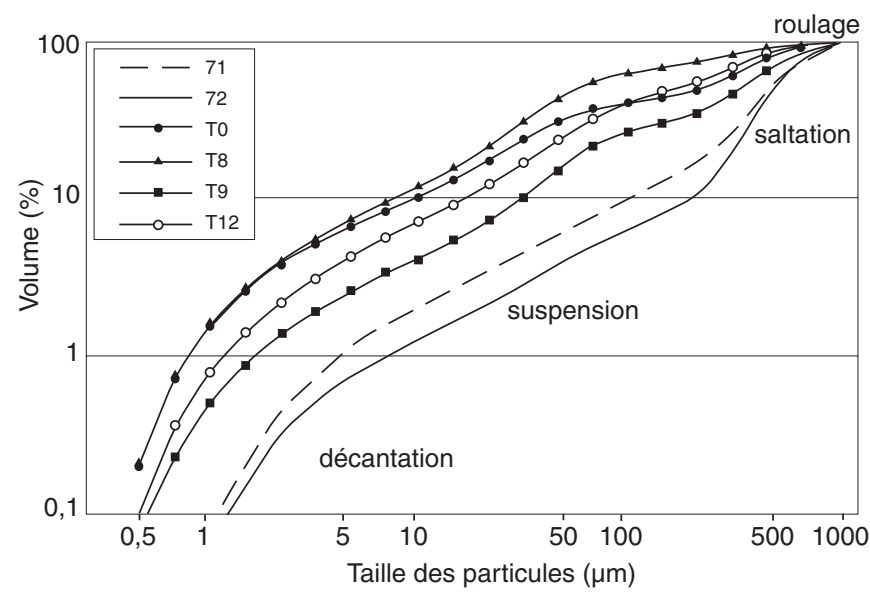

FIGURE 3. Courbes granulométriques cumulatives à ordonnée de probabilité des dépôts de la dépression (voir fig. 1 et tabl. I pour la localisation des échantillons).

Grain-size distributions (cumulated curves with logarithmic ordinate scale) of the depression deposits (samples are located on Fig. 1 and Table I).

la moyenne terrasse de la Marne. Juvigné (1991) a montré que les projections éoliennes des volcans du Massif central et de l'Eifel ont atteint à plusieurs reprises le centre du Bassin parisien. Toutefois, la taille des augites peut renseigner sur leur origine. Toujours inférieures à $200 \mu \mathrm{m}$, celles de la Marne et de l'est de la dépression ont pu être apportées par le vent; par contre, celles de la Seine et de Bobigny sont plus hétérométriques et quelques-unes dépassent $400 \mu \mathrm{m}$, ce qui exclut pour ces dernières un transport aérien et impose une origine fluviatile (Tourenq et Pomerol, 1995). La découverte de ces augites de grande taille à Villemomble, à Rosny et à Bobigny donne en outre un nouvel indice pour le tracé du «fleuve à augite » qui passait à l'est de Paris et non au sud-ouest comme le suggéraient Tourenq et Pomerol (1995).
Les profils longitudinaux des terrasses de la dépression montrent, entre le Bois de la Tussion et Saint-Denis, une pente forte, peu différente de celle des petits cours d'eau actuels mais beaucoup trop importante pour correspondre au passage d'une rivière comme la Marne (fig. 4). Si cette dernière était tout de même passée par là, les dépôts conservés ne pourraient pas être originels, ils auraient forcément été remaniés par le travail des petits affluents de la Seine. II apparaît donc que les dépôts de la dépression ne sont pas des alluvions en place de la Marne ni de la Seine. En revanche, la Beuvronne a pu emprunter la dépression et rejoindre la Seine qui empruntait le seuil de Gagny situé à $60 \mathrm{~m}$ d'altitude: la finesse des alluvions et la présence de grenat et de hornblende tendent à prouver de tels apports. La capture de la Beuvronne par recul de tête d'un affluent de la Marne passant par Claye-Souilly a pu se produire après l'accumulation des alluvions de la dépression, ainsi que le suggérait Dollfus (1903). La pente anormalement forte du cours aval de la Beuvronne est un argument supplémentaire à cette capture. Cela indique aussi que ce cours d'eau n'a pas régularisé son profil longitudinal depuis cette capture ancienne, bien que le creusement post-glaciaire atteigne plusieurs mètres sous l'étiage actuel. Ensuite, le remblaiement holocène s'est effectué en quatre phases successives datées par des données archéologiques de la période atlantique, du Subboréal, du Subatlantique et après l'époque romaine (Lebret et Halbout, 1991 ; Le Béchennec et al., 1998; Orth et al., 2004).

Les dépôts conservés à Bobigny sont plus récents en raison de leur moindre altération et de leur plus faible altitude (42 $\mathrm{m}$ ). Ils se raccordent avec la terrasse saalienne de la Seine à Saint-Denis, et traduisent deux séquences de mise en place. Les matériaux inférieurs, plus grossiers et affectés de quelques figures de gel, ont subi des conditions climatiques périglaciaires dont l'âge reste incertain: saalien ou weichsélien (Roblin-Jouve, 1995). Les formations supérieures, plus fines et plus riches en augite, pourraient correspondre à des
O Confluence Seine-Marne

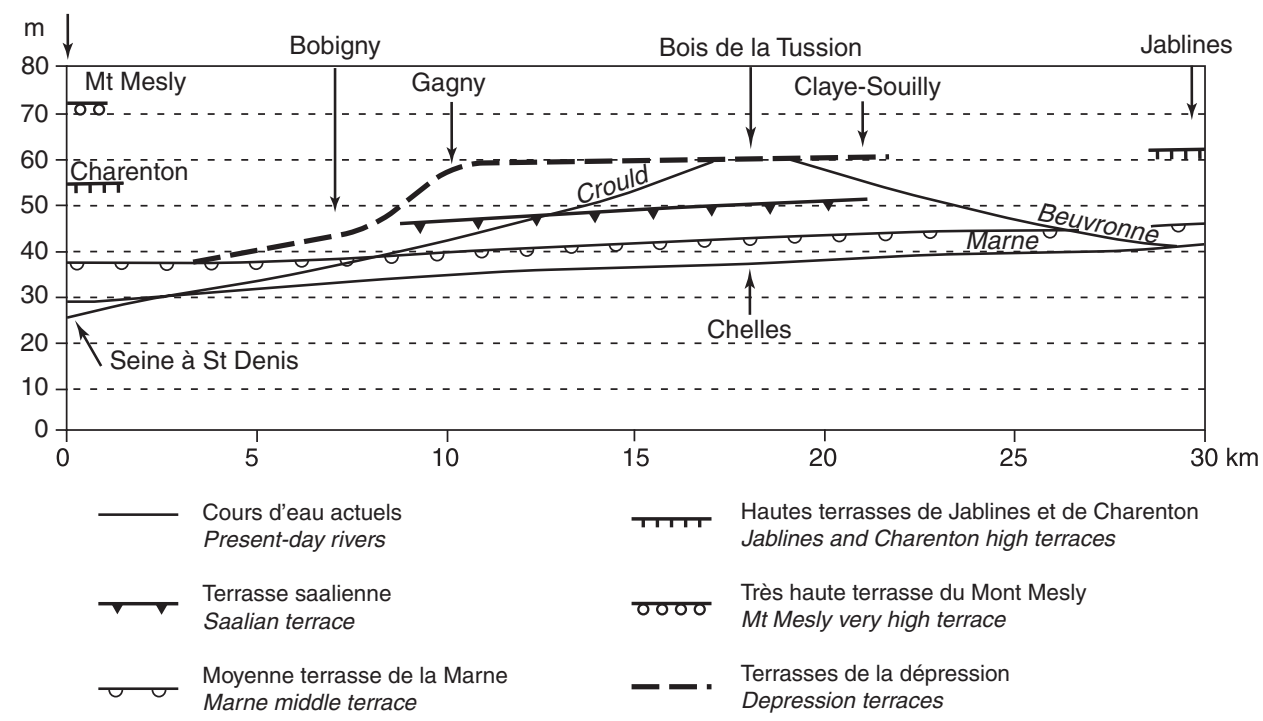

FIGURE 4. Profils longitudinaux des cours d'eau, des terrasses de la Marne et de la Seine et des terrasses de la dépression de ClayeSouilly-Saint-Denis.

Longitudinal profiles of the rivers, the Seine and Marne terraces and the terraces in the ClayeSouilly-Saint-Denis depression. 
débordements tardiglaciaires. Des saupoudrages éoliens, comme ceux du Tardiglaciaire reconnus par Juvigné (1991) et Étienne et Larue (1996) dans le centre du Bassin parisien, semblent indispensables pour expliquer les $24 \%$ d'augite de l'échantillon 73.

\section{RÔLE DES PHÉNOMĖNES DE DISSOLUTION}

La dissolution du gypse et du calcaire de Saint-Ouen et les effondrements qui en résultent contribuent à une exportation de matériau vers les vallées principales, mais des transports fluviaux sont nécessaires pour expliquer le dégagement de la dépression par érosion différentielle avant la mise en place des alluvions anciennes. Dollfus (1903) a observé entre Sevran et Aulnay un effondrement elliptique de $15 \mathrm{~m}$ sur $12 \mathrm{~m}$ et de 15-17 $\mathrm{m}$ de profondeur, qui s'est rempli d'eau jusqu'à $-1,50 \mathrm{~m}$. Des entonnoirs identiques, appelés torrents ou bouillons, se sont produits en 1685 et 1858, sur la rive droite du canal de l'Ourcq. Récemment, le Bois de la Tussion a dû être interdit aux promeneurs suite à des effondrements identiques. Tous ces effondrements jalonnent l'axe de la dépression et résultent de la dissolution du substrat gypseux et calcaire et de son évacuation, non par "une rivière cachée» selon Dollfus (1903), mais plutôt par la nappe souterraine s'écoulant vers la Seine.

Par ailleurs, les extrémités de cette dépression ont pu être envahies par les eaux de la Marne et de la Seine, à la faveur de crues. Lors des crues historiques de la Seine, Wyss (1996) signale que les eaux de cette dernière repoussaient celles du Crould et inondaient le secteur de Saint-Denis. Ces entrées pourraient expliquer localement la présence de minéraux spécifiques aux deux cours d'eau et également la régularisation topographique des creux engendrés par les effondrements.

\section{CONCLUSION}

Ainsi, les dépôts anciens de la dépression sont constitués essentiellement d'alluvions et d'apports locaux mêlés à des retombées éoliennes de projections volcaniques. Dans la partie orientale, il s'agit surtout d'alluvions de la Beuvronne déposées avant sa capture par la Marne et de colluvions issues du massif de l'Aulnay. Cette capture a permis la conservation vers $60 \mathrm{~m}$ d'altitude de ces dépôts contemporains de la haute terrasse de Jablines. Dans le secteur de Bobigny, il s'agit surtout d'alluvions des affluents du Crould qui remanient des apports plus anciens de la Seine; leur altitude plus faible (42 $\mathrm{m}$ ) est due au déblaiement effectué par le réseau du Crould en fonction de l'évolution du niveau de base de la Seine à Saint-Denis. Ces alluvions, plus récentes, se raccordent à la terrasse saalienne de la Seine. La présence d'augites supérieures à $400 \mu \mathrm{m}$ de diamètre dans les seuils de Gagny-Villemomble et de Rosny témoigne du passage d'une paléo-Seine, le "fleuve à augite ", au Pléistocène inférieur. Le creusement de la dépression apparaît donc plus ancien dans la partie orientale, puisque antérieur à l'accumulation des hautes terrasses, que dans la partie occidentale où il s'est poursuivi jusqu'au Saalien. Des datations des alluvions de la dépression permettraient probablement de mieux préciser.
La conjonction de plusieurs processus dans le façonnement de la dépression, tels les effondrements d'origine karstique, rend délicate une reconstitution plus fine.

\section{REMERCIEMENTS}

Nous remercions R. Étienne (Laboratoire rhodanien de Géomorphologie) pour la détermination des minéraux lourds, A.M. Mercier (Université du Maine) pour l'analyse des minéraux argileux, M. Marandola (Université Paris XII-Val de Marne) pour la réalisation des figures, $M$. Church, $B$. Hétu et P.J.H. Richard pour leurs critiques et leurs suggestions qui ont permis l'amélioration du manuscrit.

\section{RÉFÉRENCES}

Bishop, P., 1995. Drainage rearrangement by river capture, beheading and diversion. Progress in Physical Geography, 19: 449-473.

Bourdier, F., 1969. Étude comparée des dépôts quaternaires des bassins de la Seine et de la Somme. Bulletin d'information de l'Association des Géologues du Bassin de Paris, 21 : 169-220.

Cailleux, A., 1943. Les alluvions anciennes de la Seine et la Marne au voisinage du confluent. Bulletin des Services de la Carte Géologique de France, 212, $44: 39-61$.

Caudron, M., Labourguigne, J. et Putallaz, J., 1971. Carte géologique, feuille (184) Lagny, notice explicative. Bureau de Recherches Géologiques et Minières, Orléans, $44 \mathrm{p}$.

Cavelier, C. et Lorenz, J., 1987. Aspect et évolution géologiques du Bassin parisien. Bulletin d'information des Géologues du Bassin de Paris, Mémoire hors série 6, $271 \mathrm{p}$.

Chaput, E., 1923. Recherches sur les terrasses alluviales de la Seine entre la Manche et Montereau. Bulletin des Services de la Carte Géologique de France, 153, $27: 281-420$.

Davis, W.M., 1895. La Seine, la Meuse et la Moselle. Annales de Géographie, 19: $25-49$.

Diffre, P., 1980. Carte géologique Paris et sa proche banlieue à 1/25 000, feuille Paris-est, notice explicative. Bureau de Recherches Géologiques et Minières, Orléans, $31 \mathrm{p}$.

Dollfus, G.F., 1903. Sur les effondrements de la plaine de Sevran. Comptes Rendus de l'Académie des Sciences, Paris, 137, p. 279-281.

Durbet, G., Rodriguez, P., Badalian, L., Djillali, H., Gauthier, A., Laurent, M. Ricard, J.L. et Wattez, J., 1997. Découverte d'un site paléolithique moyen dans les alluvions saaliennes du confluent Seine-Marne à Maisons-Alfort (Val-de-Marne). Comptes Rendus de l'Académie des Sciences, Paris, 324, p. 505-512.

Étienne, R. et Larue, J.P., 1996. Mise en évidence d'une troisième source de clinopyroxènes dans les alluvions de la Seine et de ses affluents méridionaux. Comptes Rendus de l'Académie des Sciences, Paris, 323, p. 849854.

Hadjouis, A., 2001. Analyse sédimentaire complémentaire de la ZAC d'Alfort I, Maisons Alfort (Val de Marne). Apport des minéraux lourds. Rapport d'étude, Laboratoire départemental d'archéologie, Conseil Général du Val de Marne, Villejuif, 48 p.

Harmand, D., Weisrock, A., Gamez, P., Le Roux, J., Occhietti, S., Deshaies, M., Bonnefont, J.C. et Sary, M., 1995. Nouvelles données relatives à la capture de la Moselle. Revue de Géographie de l'Est, 3-4: 321-343.

Hirschauer, A., 1991. Les bassins de retenue d'eau pluviale de la Seine-SaintDenis: incidences de l'environnement géotechnique sur leur conception. Mémoire d'ingénieur CNAM, Conseil Général de Seine-Saint-Denis, SaintDenis, 229 p. + annexes.

Juvigné, E., 1991. Distribution de vastes retombées volcaniques originaires de l'Eifel et du Massif Central aux temps post-glaciaires dans le NE de la 
France et les régions voisines. Comptes Rendus de l'Académie des Sciences, Paris, 312, p. 415-420.

Lafarge, I.,1998. Le peuplement dans le nord-est du Bassin parisien de la Tène au Xle siècle: la vallée du Ru de Montfort (Aubervilliers, Bobigny, Le Bourget, La Courneuve, Drancy, Pantin, Seine-Saint-Denis). Mémoire de Maîtrise, Université de Paris VIII, $168 \mathrm{p}$.

Larue, J.P., 2003. L'encaissement inégal de la Seine et la Loire dans le Bassin parisien (France). Géographie physique et Quaternaire, 57:21-36.

Larue, J.P. et Étienne, R., 1997. Les changements de cours de la Sarthe entre Le Mans et Sablé-sur-Sarthe (France). Géographie physique et Quaternaire, $51: 285-294$

Lebret, P. et Halbout, H., 1991. Le Quaternaire dans le Val d'Oise. Centre de Géomorphologie de Caen, Caen, Bulletin 39-40, 267 p.

Le Béchennec, Y., Marion, S., Frère, S., Mistrot, V., Munoz, C et Roblin-Jouve, A., 1998. Bobigny, La Vache-à-l'aise (Seine-Saint-Denis). Conseil Général de Seine-Saint-Denis, Saint-Denis, rapport de fouille de sauvetage, p. 17 26.

Lécolle, F., 1989. Le cours moyen de la Seine au Pléistocène moyen et supérieur. Géologie et préhistoire. Thèse de doctorat d'État, Université Paris VI, Rouen, 549 p.

Leroyer, C., Pastre, J.F., Fontugne, M. et Limondin-Lozouet, N., 1997. Le Tardiglaciaire et le début de l'Holocène dans le bassin aval de la Marne (Seine et Marne, France) : chronostratigraphie et environnement des occupations humaines, p. 151-164. In Le Tardiglaciaire en Europe du nord-ouest. Actes du $119^{\circ}$ congrès national des sociétés historiques et scientifiques, Amiens, $625 \mathrm{p}$.

Losson, B. et Quinif, Y., 2001. La capture de la Moselle: nouvelles données chronologiques par datations U/Th sur spéléothèmes. Karstologia, 37 : 2940.

Maher, E., Harvey, A.M., Derek, F., 2007. The impact of a major Quaternary river capture on the alluvial sediments of a beheaded river system, the Rio Alias SE Spain. Geomorphology, 84 : 344-356.

Mather, A.E., 2000. Adjustment of a drainage network to capture induced baselevel change: an example from the Sorbas Basin, SE Spain. Geomorphology, 34: 271-289.

Michel, J.P., 1972. Le Quaternaire de la région parisienne. Thèse de doctorat d'État, Université Paris VI, 572 p.

Moore, D.M. et Reynolds, R.C., 1997. X-Ray Diffraction and the Identification and Analysis of Clay Minerals. Oxford University Press, Oxford, 378 p.

Orth, P., Pastre, J.F., Gauthier, A., Limondin-Lozouet, N. et Kunesch, S., 2004. Les enregistrements morphosédimentaires et biostratigraphiques des fonds de vallée du bassin versant de la Beuvronne (Bassin parisien, Seine-etMarne, France) : perception des changements climato-anthropiques à l'Holocène. Quaternaire, $15:$ 285-298.

Parfenoff, A., Pomerol, C. et Tourenq, J., 1970. Les minéraux en grains, méthodes d'étude et détermination. Masson, Paris, $578 \mathrm{p}$.
Pastre, J.F. et Cantagrel, J.M., 2001. Téphrostratigraphie du Mont Dore (Massif central, France). Quaternaire, 12:249-267.

Pastre, J.F. et Leroyer, C., 1995. Les deux captures du système Marne-GrandMorin (Brie septentrionale, France). Colloque Paléoréseaux hydrographiques quaternaires, Nancy, résumés, p. 73.

Pastre, J.F. et Leroyer, C., 1997. La capture du Grand-Morin par la Marne (Bassin parisien, France) : âge et mécanisme. Géographie physique et Quaternaire, 51:347-350.

Pastre, J.F., Fontugne, M., Kuzucuoglu, C., Leroyer, C., Limondin-Lozouet, N., Talon, M. et Tisnerat, N., 1997. L'évolution tardi- et post-glaciaire des lits fluviaux au NE de Paris (France). Relations avec les données paléoenvironnementales et l'impact anthropique sur les versants. Géomorphologie, 4 : 291-312.

Pomerol, C., 1967. Esquisse paléogéographique du Bassin de Paris à l'ère tertiaire et aux temps quaternaires. Revue de Géographie physique et de Géologie dynamique, 9:55-85.

Pomerol, C. et Feugueur, L., 1974. Bassin de Paris, Île de France, Pays de Bray. Collection des Guides géologiques régionaux, Masson, Paris, $216 \mathrm{p}$.

Roblin-Jouve, A., 1980. Le paysage paléolithique de la vallée de la Seine de Corbeil à Bray-sur-Seine. Thèse de doctorat $3^{e}$ cycle, Université Paris VII, 2 tomes, $278 \mathrm{p}$.

Roblin-Jouve, A., 1995. Esquisse géomorphologique du département de SeineSaint-Denis, $10 \mathrm{p}$.

Roblin-Jouve, A. et Rodriguez, P., 1997. Paléogéographie des occupations humaines du centre du Bassin parisien à partir du Tardiglaciaire, p. 141150. In Le Tardiglaciaire en Europe du nord-ouest. Actes du $119^{\circ}$ congrès national des sociétés historiques et scientifiques, Amiens, $625 \mathrm{p}$.

Rodriguez, P., 1994. Maisons Alfort, ZAC d'Alfort I, étude géomorphologique. Laboratoire départemental d'archéologie du Val de Marne, Villejuif, 124 p. + annexes.

Soyer, R., 1953. Géologie de Paris. Mémoire pour Servir à l'Explication de la Carte Géologique de France. Imprimerie Nationale, Paris, p. 561-582.

Tourenq, J. et Pomerol, C., 1995. Mise en évidence, par la présence d'augite du Massif central, de l'existence d'une pré-Loire-pré-Seine coulant vers la Manche au Pléistocène. Comptes Rendus de l'Académie des Sciences, Paris, 320, p. 1163-1169.

Tricart, J., 1952. La partie orientale du Bassin de Paris. Étude morphologique. Sedes, Paris, $467 \mathrm{p}$.

Visher, G.S., 1969. Grain size distributions and depositional processes. Journal of Sedimentary Petrology, 39: 1074-1106.

Weisrock, A., 1997. Avant-propos. Cent ans après Davis: la question des captures et les paléoréseaux hydrographiques quaternaires d'après les exemples de l'Europe du Nord-Ouest. Géographie physique et Quaternaire, $51: 261-266$.

Wyss, M.,1996. Atlas historique de Saint-Denis, des origines au XVIII ${ }^{\mathrm{e}}$ siècle. Maison des Sciences de l'Homme, Paris, $444 \mathrm{p}$. 